\title{
Short-time repeat high-risk HPV testing by self-sampling for screening of cervical cancer
}

\author{
U Gyllensten ${ }^{*, 1,3}$, K Sanner ${ }^{2,3}$, I Gustavsson', M Lindell', I Wikström² and E Wilander' \\ 'Department of Immunology, Genetics and Pathology, Rudbeck Laboratory, SciLifeLab, Uppsala University Hospital, Uppsala, Sweden; ${ }^{2}$ Department of \\ Women's and Children's Health, Uppsala University, Uppsala University Hospital, Uppsala, Sweden
}

BACKGROUND: Testing for high-risk human papillomavirus (HPV) in primary screening for cervical cancer is considered more sensitive, but less specific, in comparison with Pap-smear cytology. Women with persistent HPV infections have a higher risk of developing cervical intraepithelial neoplasia $2+(\mathrm{CIN2}+)$ lesions. This study was performed to evaluate the gain in specificity for detection of histologically confirmed CIN2 + lesions achieved by short-time repeat testing for high-risk HPV in women aged 30-65 years, with the primary sample for HPV analysis taken by self-sampling.

METHODS: A total of 8000 women in Uppsala County, aged 30-65 years, who had not attended organised screening for 6 years or longer, were offered self-sampling of vaginal fluid at home and the samples sent for HPV typing. Of these, 8\% (669) were not possible to contact or had performed hysterectomy. Women positive for high-risk HPV in the self-sampling test were invited for a follow-up HPV test and a cervical biopsy on average 3 months after the initial HPV test.

RESULTS: In all, 39\% (2850/733I) of invited women chose to perform self-sampling of vaginal fluid at home. High-risk HPV infection was found in 6.6\% (188) of the women. In all, $89 \%$ of the women testing HPV positive performed a follow-up examination, on average 2.7 months, after the first test and $59 \%$ of these women were HPV positive in the follow-up test. The prevalence of CIN2 + lesions in women with an initial HPV-positive test was $23 \%(95 \% \mathrm{Cl} 18-30 \%)$ and in women with two consecutive HPV-positive tests was $41 \%$ (95\% Cl $31-51 \%)$. In women with two positive HPV tests, the prevalence of CIN2+ lesions varied from $49 \%$ in women at age 30-39 years to 24\% in women at age 50-65 years. Short-time repeat HPV testing increased the specificity for detection of CIN2 + lesions from about $94.2 \%$ to $97.8 \%$. The most prevalent HPV types were HPV I6 (32\%), followed by HPV I 8/45 (19\%) and HPV 33/52/58 (19\%).

CONCLUSION: The short-time persistence of high-risk HPV infection in this age group was about 60\%. Repeat testing for high-risk HPV using self-sampling of vaginal fluid can be used to increase the specificity in the screening for cervical cancer in women aged 30-65 years.

British Journal of Cancer (20II) I 05, 694-697. doi:I0.1038/bjc.20 I I.277 www.bjcancer.com

Published online 2 August 2011

(C) 20II Cancer Research UK

Keywords: cervix; screening; carcinoma; HPV test; persistence; self-sampling

The introduction of organised cytological screening in Sweden in the late 1960s has resulted in a decrease of the prevalence of cervical cancer by $\sim 50 \%$ (Bergstrom et al, 1999). However, not all women have attend the organised screening programme and a large fraction of cervical cancers occur in women who have not participated in the cytological screening (Andrae et al, 2008). By offering self-sampling at home, the population coverage of the screening can be improved (Sanner et al, 2009; Gok et al, 2010).

Infection with high-risk types of human papillomavirus (HPV) is the dominant risk factor for development of cervical carcinoma (zur Hausen, 1991), and it has been shown that testing for highrisk HPV infection can be used as an adjunct test or an alternative to Pap-smear screening (Wright et al, 2004; Cuschieri and Cubie,

\footnotetext{
*Correspondence: Professor U Gyllensten;

E-mail: ulf.gyllensten@igp.uu.se

${ }^{3}$ These authors contributed equally to this work.

Received 27 April 201 I; revised 23 June 2011; accepted 27 June 201।; published online 2 August 2011
}

2005; Meijer et al, 2009). Testing for high-risk HPV in primary screening has also been shown to be twice as sensitive as Pap-smear screening for detection of premalignant (cervical intraepithelial neoplasia $2+$; CIN2 + ) cervical lesions (Ronco et al, 2010). In postmenopausal women, the difference in sensitivity between cytology and HPV typing may be even larger, with testing for high-risk HPV being three times more sensitive than cytological screening for detecting women at risk for tumour development (Gyllensten et al, 2010).

The use of testing for high-risk HPV in primary screening is complicated by the low specificity of a single HPV-positive test as an indictor of cervical cancer risk. Many women have transient infections, and HPV testing in primary screening could therefore result in inconvenience if no premalignant cell alterations are diagnosed in the cytological smear and no treatment is available for HPV infection. The value of HPV testing can be increased by triaging with cytology or using p16 staining. However, women with a persistent HPV infection have been shown to be at higher risk of developing cervical cancer (Koshiol et al, 2008), indicating that follow-up by repeat typing of HPV could be used to identify women at risk. 
Previous studies have evaluated the use of repeated HPV testing when the samples were taken in a clinical setting. In this study, we have evaluated the use of short-time repeat testing for high-risk HPV to identify histological CIN2 + lesions on the cervix, when the first sample for HPV analysis is obtained by self-sampling at home. If short-time repeat testing in combination with selfsampling can be shown to efficiently improve the specificity of testing positive for high-risk HPV, only women with two consecutive positive HPV tests need to be admitted to gynaecological surgery for follow-up and examined by colposcopy for the presence of histological CIN2 + lesions on the cervix.

\section{MATERIAL AND METHODS}

During 2008-2010, a total of 8000 women (3000 in 2008 and 2500 per year during 2009-2010) from Uppsala County, between the ages of 30-65 years and who had not attended organised cytological screening for 6 years or longer according to the data registry of the organised screening, were offered self-sampling of vaginal fluid at home using the Qvintip device (Aprovix $A B$, Uppsala, Sweden; Stenvall et al, 2006; Sanner et al, 2009). Among the 8000 women invited, 669 were not possible to reach because of inaccurate address information or had a previous operation with hysterectomy.

Of the remaining women $(n=7331), 2850(39 \%)$ chose to participate and sent a vaginal fluid sample to Aprovix for high-risk HPV analysis. The samples were analysed using the hybrid capture II (HC2) method, which detects the presence of any of 13 high-risk HPV types $(16,18,31,33,35,39,45,51,52,56,58,59$ and 68). The result of the HPV analyses was communicated to the participating women within 2-3 weeks after the arrival of the vaginal sample to the laboratory. Women testing positive for HPV were offered an attendance to a gynaecological surgery for a follow-up examination within 1-3 months. At the follow-up visit, the physician performed a colposcopy, collected biopsy samples from the portio (directed biopsy samples or four biopsy samples from the transformation zone if the portio appeared normal) and sampled cervical fluid for a repeat HPV analysis. The second HPV test was performed using the hpVIR method, which detects the following high-risk HPV types $16,18,31,33,35,39,45,51,52,56$, 58 and 59 (18 and 45 are detected together, and 33, 52 and 58 as one group; Gustavsson et al, 2009a, b). Women with two consecutive positive HPV tests, and either abnormal cytology (ASCUS-CIN3) and/or CIN1-3 at the histopathological analysis, were recommended a further follow-up, or in the case of CIN2+ in the biopsy sample or Pap smear, were treated with surgical cone resection. The cervical biopsy samples were examined by specialists in surgical pathology. When the woman was $>30$ years old, diagnostic conisation was performed also for women with CIN 1 histopathology in cases when the transformation zone not was visualised. The end point of the study was identification of a $\mathrm{CIN} 2+$ in the cervical biopsy or cone resection. The CIN2+ corresponds to the high-grade squamous intraepithelial lesion
(HSIL) according to the Bethesda definition of premalignant cell alterations on the cervix.

\section{RESULTS}

A total of $39 \%$ (2850/7331) of participating women performed selfsampling of vaginal fluid at home. No difference in attendance rate was seen for different age groups (data not shown). In all, $6.6 \%$ $(188 / 2850)$ of the participants were positive for high-risk HPV in the self-sample. The prevalence of high-risk HPV decreased with age, from $11.5 \%$ in women aged $30-39$ years to $4.7 \%$ in women aged 50-65 years (Table 1 ). The CIN2 + occurred in 44 of the HPV-positive women $(23 \%, 95 \%$ CI $18-30 \%)$. The prevalence of CIN2 + among HPV-positive women was $33 \%$ in women aged 30-39 years and $14 \%$ in women aged $50-65$ years (Table 1 ).

Of the 188 women that were positive for high-risk HPV in the self-sample, 168 performed a follow-up examination, giving a compliance of $89 \%(168 / 188)$. Eleven of the women chose to visit a midwife reception for sampling of cervical smear for cytological screening and HPV analysis. These women are not further described. In three additional women, cervical biopsy samples were collected but no sample for HPV analysis was obtained, and these were also excluded from further analyses.

In the remaining 154 women, cervical biopsies were performed in combination with sampling for a repeat HPV testing at a gynaecological surgery. Five HPV tests were regarded as invalid because of low amount of cells in the sample. In the remaining 149 women with two valid consecutive high-risk HPV tests, of which 88 were positive and 61 negative, the presence of CIN2+ lesions could be evaluated. The mean time between the first and second HPV test was 2.7 months, ranging from 4.0 months for women in the age group of 30-39 years to 1.9 months for women in the age group of $50-65$ years.

Repeated HPV positivity was found in $67 \%$ of women of age $30-39$ years, in $64 \%$ in women of age $40-49$ years and in $47 \%$ of women of age 50-65 years (Table 2). The difference between age groups was not statistically significant $(P=0.350)$. Among the 88 women with a persistent HPV infection, $36(41 \%, 95 \%$ CI $31-51)$ had histologically defined CIN2 + lesions (Table 2). The prevalence of CIN2 + in women with a short-time persistent HPV infection varied from $49 \%$ in women of age $30-39$ years to $24 \%$ in women of age $50-$ 65 years (Table 2). Among women that were HPV positive in their first test, $23 \%$ had CIN2 +, whereas $41 \%$ of women with two consecutive positive HPV tests had a CIN2+.

Information on the infecting HPV type was obtained for 88 women participating in the second HPV test. HPV16 was the most prevalent type (32\%), followed by HPV18/45 (19\%) and the group HPV33/52/58 (19\%). Among other types, HPV31 occurred in 9\%, HPV 35 in 3\%, HPV 39 in 3\%, HPV51 in 3\%, HPV6 in $4 \%$ and HPV59 in $10 \%$ of women positive in the second HPV test. The prevalence of HPV16 decreased with age, from $34 \%$ in premenopausal women to $23 \%$ in postmenopausal ( $\geqslant 50$ years old) women $(P<0.02)$. The specificity of using a single HPV test in screening in

Table I Prevalence of infection with high-risk HPV in primary screening and histological CIN2+ and CIN3 lesions in HPV-positive women in relation to age in women performing self-sampling of vaginal fluid at home

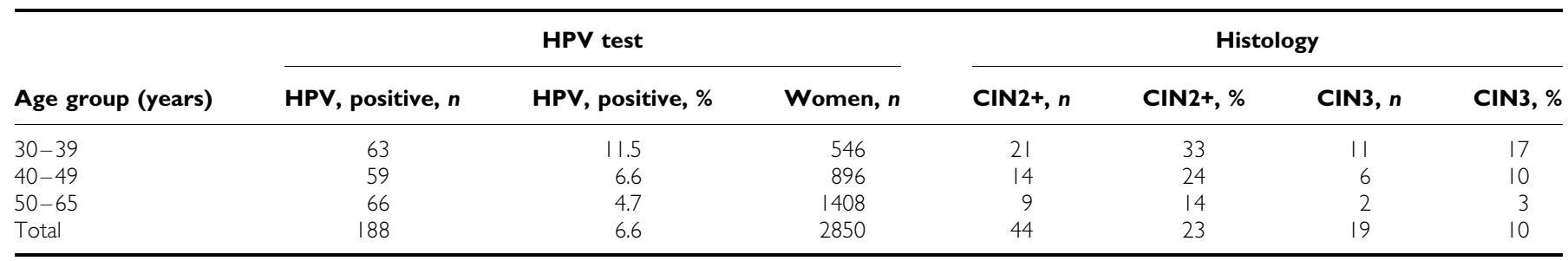

Abbreviations: $\mathrm{CIN}=$ cervical intraepithelial neoplasia; HPV = high-grade squamous intraepithelial lesion. 
Table 2 Prevalence of infection with high-risk HPV in two consecutive tests and of histological CIN2+ and CIN3 lesions in women in different age categories

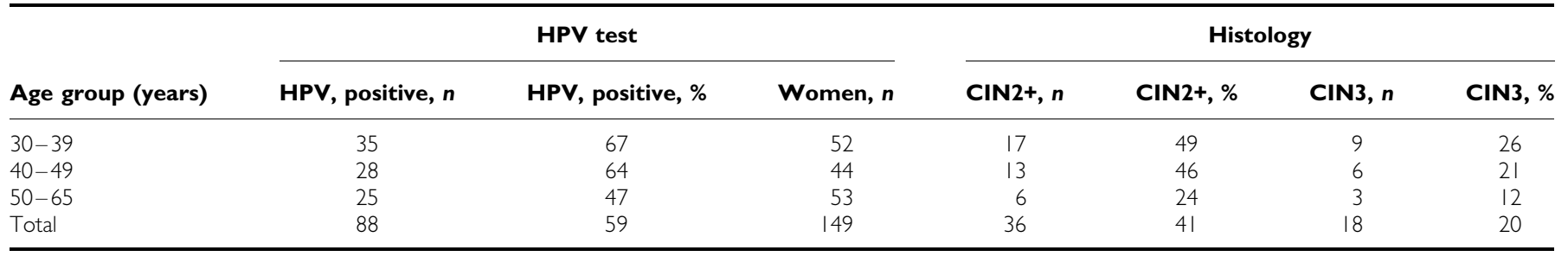

Abbreviations: $\mathrm{CIN}=$ cervical intraepithelial neoplasia; $\mathrm{HPV}=$ high-grade squamous intraepithelial lesion.

Table 3 Specificity (\%) for identification of histological CIN2+ lesions in relation to age in women with a high-risk HPV infection in primary screening and in women with two short-time consecutive positive HPV tests

\begin{tabular}{lcc}
\hline Age group (years) & Primary test positive & Repeat positive \\
\hline $30-39$ & 92 & 96.6 \\
$40-49$ & 94.9 & 98.2 \\
$50-65$ & 95.6 & 98.4 \\
Mean value & 94.2 & 97.8 \\
\hline
\end{tabular}

Abbreviations: $\mathrm{CIN}=$ cervical intraepithelial neoplasia; $\mathrm{HPV}=$ high-grade squamous intraepithelial lesion.

comparison with two consecutive HPV tests for detection of histologically defined CIN2 + is shown in Table 3. A single highrisk HPV test in primary screening showed a specificity of $94.2 \%$, whereas applying a repeat high-risk HPV test increased the specificity to $97.8 \%$. The specificity of both primary and repeat high-risk HPV testing increased with age.

\section{DISCUSSION}

In Uppsala County, the coverage in the organised cytological screening is $<70 \%$ and low in comparison with other counties in Sweden. This was one of the main reasons for introducing selfsampling of vaginal fluid at home in combination with HPV testing as an adjunct in the screening for cervical cancer in 2007 (Sanner et al, 2009). The low coverage in the Uppsala County is one possible explanation for the relatively high acceptance rate for selfsampling of vaginal fluid at home (39\%) in comparison with other similar studies (Gok et al, 2010).

The organised screening in Sweden has reduced the prevalence of cervical cancer from about 1000 cases per year to around 450 cases per year (Bergstrom et al, 1999). In a recent nationwide audit in Sweden, it was reported that $65 \%$ of women with cervical cancer have not participated in the screening, whereas around $25 \%$ of the cervical carcinomas develop among women who regularly have attended the screening and have been repeatedly informed that their smear is normal (Andrae et al, 2008). This observation emphasises the need to develop new means of increasing the participation rate and obtain novel screening methods with higher sensitivity than Pap-smear cytology (Sanner et al, 2009; Gok et al, 2010).

Testing for high-risk HPV in primary screening results in a higher sensitivity in comparison with Pap-smear screening (Wright et al, 2004; Cuschieri and Cubie, 2005; Meijer et al, 2009). HPV testing identifies around twice as many CIN2 + lesions per 1000 examinations as the regular Pap-smear screening (Ronco et al, 2010). The difference between HPV typing and cytology seems to increase with age (Gyllensten et al, 2010). An introduction of HPV testing in primary screening is facilitated by its high sensitivity (Wright et al, 2004; Cuschieri and Cubie, 2005; Meijer et al, 2009) and by its high negative predictive value, indicating that screening intervals can be prolonged (Naucler et al, 2007; Kitchener et al, 2009), but it has the disadvantage of a low specificity (Ronco et al, 2010). A number of studies have shown that repeat testing for HPV has a higher specificity than a single test in primary screening (Plummer et al, 2007; Shiffman and Smith, 2007; Rodriguez et al, 2008; Castle et al, 2009). Further, women with aberrant cytology have a lower clearance rate of highrisk HPV in comparison with women showing normal cytology (Bulkmans et al, 2007). A meta-analysis showed that persistent HPV infection is consistently associated with higher risk of CIN2 + /HSIL (Koshiol et al, 2008).

By repeating the HPV test within a few months of the primary test, and performing clinical follow-up only on women with a short-time persistent HPV infection, we obtained a higher specificity for the entire age spectrum of women 30-65 years of age in comparison with primary high-risk HPV testing. The time interval between the initial and repeat HPV test was chosen to be similar to that used in the follow-up of women with ASCUS and CIN1 alterations in the routine cytological screening. As transient HPV infections are known to remain for 6-24 months, some of the women testing positive in the two subsequent HPV tests are likely to have cleared the virus later. Thus, our study design will underestimated the specificity of repeat HPV testing. However, as information of a high-risk HPV infection may cause discomfort to the women, a reduced length of the time period between samplings was chosen.

We note that there is a lower prevalence of CIN2 + in women with short-time persistent HPV infections in the higher age group. This could be due to the shorter follow-up time in the age group of $50-65$ years ( 1.9 months) relative to the women aged $30-39$ years (4 months). Another explanation is that the transformation zone between squamous and glandular mucosa moves upwards in the cervical canal after menopause and it is possible that a number of $\mathrm{CIN} 2+$ lesions are not detected in older women despite gynaecological examination, colposcopy and cervical biopsy.

We used two different high-risk HPV tests in the present investigation. Although the hpVIR test is favoured by its ability to be type specific, the self-sampling device Qvintip has been validated in combination with the FDA-approved HC2 method (Stenvall et al, 2007; Sanner et al, 2009). The two methods give similar but not absolutely identical results with regard to the identification of smears harbouring high-risk HPV (Gustavsson et al, 2009a). The hpVIR assay does not include HPV 68. The lack of HPV68 in the hPVIR assay could result in women classified as having a transient rather than persistent infection. This would reduce the apparent value of repeat typing and lead to an underestimation of the specificity. Given the relatively low frequency of this HPV type, the effect is likely to be rather small. Studies are in progress to introduce hpVIR in combination with self-sampling of vaginal fluid at home (Gustavsson et al, 2009b).

As expected, HPV 16 and 18/45 were the most common virus types present, together found in $51 \%$ of the HPV-positive samples, 
followed by the group HPV33/52/58 (19\%). The prevalence of HPV16 decreased with age from $34 \%$ in premenopausal women to $23 \%$ in postmenopausal women. This observation may have some significance with respect to the long-term effect of human HPV vaccination, in particular for countries with an organised screening, in which most cervical cancer cases occur in women 50 years of age and older.

\section{REFERENCES}

Andrae B, Kemetli L, Sparen P, Silfverdal L, Strander B, Ryd W, Dillner J, Tornberg S (2008) Screening-preventable cervical cancer risks: evidence from a nationwide audit in Sweden. J Natl Cancer Inst 100: 622-629

Bergstrom R, Sparen P, Adami HO (1999) Trends in cancer of the cervix uteri in Sweden following cytological screening. Br J Cancer 81: 159-166

Bulkmans NW, Berkhof J, Bulk S, Bleeker MC, van Kemenade FJ, Rozendaal L, Snijders PJ, Meijer CJ (2007) High-risk HPV type-specific clearance rates in cervical screening. Br J Cancer 96: 1419-1424

Castle PE, Rodriguez AC, Burk RD, Herrero R, Wacholder S, Alfaro M, Morales J, Guillen D, Sherman ME, Solomon D, Schiffman M (2009) Short term persistence of human papillomavirus and risk of cervical precancer and cancer: population based cohort study. Bmj 339: b2569

Cuschieri KS, Cubie HA (2005) The role of human papillomavirus testing in cervical screening. J Clin Virol 32: 34-42

Gok M, Heideman DA, van Kemenade FJ, Berkhof J, Rozendaal L, Spruyt JW, Voorhorst F, Belien JA, Babovic M, Snijders PJ, Meijer CJ (2010) HPV testing on self collected cervicovaginal lavage specimens as screening method for women who do not attend cervical screening: cohort study. Bmj 340: c1040

Gustavsson I, Juko-Pecirep I, Backlund I, Wilander E, Gyllensten U (2009a) Comparison between the Hybrid Capture 2 and the hpVIR real-time PCR for detection of human papillomavirus in women with ASCUS or low grade dysplasia. J Clin Virol 45: 85-89

Gustavsson I, Lindell M, Wilander E, Strand A, Gyllensten U (2009b) Use of FTA card for dry collection, transportation and storage of cervical cell specimen to detect high-risk HPV. J Clin Virol 46: 112-116

Gyllensten U, Lindell M, Gustafsson I, Wilander E (2010) HPV test shows low sensitivity of Pap screen in older women. Lancet Oncol 11: 509-510; author reply $510-1$

Kitchener HC, Almonte M, Gilham C, Dowie R, Stoykova B, Sargent A, Roberts C, Desai M, Peto J (2009) ARTISTIC: a randomised trial of human papillomavirus (HPV) testing in primary cervical screening. Health Technol Assess 13: 1-150, iii-iv

Koshiol J, Lindsay L, Pimenta JM, Poole C, Jenkins D, Smith JS (2008) Persistent human papillomavirus infection and cervical neoplasia: a systematic review and meta-analysis. Am J Epidemiol 168: 123-137

Meijer CJ, Berkhof J, Castle PE, Hesselink AT, Franco EL, Ronco G, Arbyn M, Bosch FX, Cuzick J, Dillner J, Heideman DA, Snijders PJ (2009) Guidelines for human papillomavirus DNA test requirements for

\section{ACKNOWLEDGEMENTS}

The County Council of Uppsala, the Medical Faculty of Uppsala and the Swedish Cancer Foundation supported the study. The study has been evaluated by the local ethic committee (Dnr2004: M-202 and 2009/001). EW is a minority shareholder in the company Aprovix AB, Uppsala, Sweden. primary cervical cancer screening in women 30 years and older. Int $J$ Cancer 124: $516-520$

Naucler P, Ryd W, Tornberg S, Strand A, Wadell G, Elfgren K, Radberg T, Strander B, Johansson B, Forslund O, Hansson BG, Rylander E, Dillner J (2007) Human papillomavirus and Papanicolaou tests to screen for cervical cancer. $N$ Engl J Med 357: 1589-1597

Plummer M, Schiffman M, Castle PE, Maucort-Boulch D, Wheeler CM (2007) A 2-year prospective study of human papillomavirus persistence among women with a cytological diagnosis of atypical squamous cells of undetermined significance or low-grade squamous intraepithelial lesion. J Infect Dis 195: $1582-1589$

Rodriguez AC, Schiffman M, Herrero R, Wacholder S, Hildesheim A, Castle PE, Solomon D, Burk R (2008) Rapid clearance of human papillomavirus and implications for clinical focus on persistent infections. J Natl Cancer Inst 100: $513-517$

Ronco G, Giorgi-Rossi P, Carozzi F, Confortini M, Dalla Palma P, Del Mistro A, Ghiringhello B, Girlando S, Gillio-Tos A, De Marco L, Naldoni C, Pierotti P, Rizzolo R, Schincaglia P, Zorzi M, Zappa M, Segnan N, Cuzick J (2010) Efficacy of human papillomavirus testing for the detection of invasive cervical cancers and cervical intraepithelial neoplasia: a randomised controlled trial. Lancet Oncol 11: $249-257$

Sanner K, Wikstrom I, Strand A, Lindell M, Wilander E (2009) Selfsampling of the vaginal fluid at home combined with high-risk HPV testing. Br J Cancer 101: $871-874$

Shiffman J, Smith S (2007) Generation of political priority for global health initiatives: a framework and case study of maternal mortality. Lancet 370: $1370-1379$

Stenvall H, Wikstrom I, Backlund I, Wilander E (2007) Accuracy of HPV testing of vaginal smear obtained with a novel self-sampling device. Acta Obstet Gynecol Scand 86: 16-21

Stenvall H, Wikstrom I, Wilander E (2006) Human papilloma virus testing of vaginal smear obtained with a novel self-sampling device. Acta Derm Venereol 86: $465-467$

Wright TC, Schiffman M, Solomon D, Cox JT, Garcia F, Goldie S (2004) Interim guidance for the use of human papillomavirus DNA testing as an adjunct to cervical cytology for screening. Obstet Gynecol 103: 304-309 zur Hausen H (1991) Human papillomaviruses in the pathogenesis of anogenital cancer. Virology 184: 9-13

This work is published under the standard license to publish agreement. After 12 months the work will become freely available and the license terms will switch to a Creative Commons Attribution-NonCommercial-Share Alike 3.0 Unported License. 